\title{
Response specific temporal expectancy: Evidence from a variable foreperiod paradigm
}

\author{
Roland Thomaschke • Andrea Kiesel • \\ Joachim Hoffmann
}

Published online: 14 July 2011

(C) Psychonomic Society, Inc. 2011

\begin{abstract}
When a stimulus-response event is frequently paired with a specific foreperiod, response performance for this event is improved after this foreperiod. This phenomenon is referred to as specific temporal expectancy. In four experiments, we investigated whether stimulus- or response-related processing benefits from specific temporal expectancy. In a speeded choice reaction task, different features of the imperative stimuli were frequently paired with foreperiods in such a way that only in some experiments were the responses also frequently paired with foreperiods. Participants revealed evidence for specific temporal expectancy when responses were frequently paired with foreperiods, but not when only the stimuli were frequently paired with foreperiods. We concluded that specific temporal expectancy affects response-related processing.
\end{abstract}

Keywords Temporal processing $\cdot$ Attention $\cdot$ Motor control

Human behavior is, to a large degree, guided by expectancies about future events. Optimized anticipative behavior requires to form expectancies about what will happen, as well as about when it will happen. The latter aspect of expectancy, commonly referred to as temporal expectancy, is currently a heavily researched area (Correa, Triviño, Pérez-Dueñas, Acosta, \& Lupiáñez, 2010b; Los \& Horoufchin, 2011;

R. Thomaschke $(\square)$

Lehrstuhl für Angewandte und Allgemeine Psychologie, Universität Regensburg,

Universitätsstraße 31,

93053 Regensburg, Germany

e-mail: Roland.Thomaschke@psychologie.uni-regensburg.de

R. Thomaschke $\cdot$ A. Kiesel $\cdot$ J. Hoffmann

Lehrstuhl für Psychologie III, Universität Würzburg,

Würzburg, Germany
Nobre \& Coull, 2010; Riehle, 2005; Steinborn, Rolke, Bratzke, \& Ulrich, 2009; Vallesi, Binns, \& Shallice, 2008). One of the most common paradigms in temporal expectancy research is the foreperiod paradigm. In the foreperiod paradigm, an imperative stimulus is preceded by a warning stimulus. The interval between warning and imperative stimulus is referred to as the foreperiod (FP; Niemi \& Näätänen, 1981). When participants expect that the imperative stimulus will occur after a specific FP, they can prepare for the imperative stimulus, and, consequently, respond to the stimulus more quickly (Woodrow, 1914).

When FPs are fixed across a block of trials, the FP is clearly predictable for the participant, resulting in a high degree of temporal expectancy. A well-established finding is that response times (RTs) increase with increasing FPs when FPs are constant over a block, but vary between blocks (MüllerGethmann, Ulrich, \& Rinkenauer, 2003; Wundt, 1874). This effect is commonly explained by a reduced precision of temporal expectancy, due to the deterioration of the accuracy of time estimation for longer time intervals (Grondin, 2001).

However, when FPs vary randomly from trial to trial, temporal expectancy is generally much weaker than in constant-FP designs, because participants can only guess when the target stimulus will appear (Awramoff, 1903; Cardoso-Leite, Mamassian, \& Gorea, 2009). In variable-FP paradigms, RTs are commonly found to decrease with increasing FPs (Näätänen, 1970; Woodrow, 1914). This is known as the variable-FP effect (Lohmann, Herbort, Wagener, \& Kiesel, 2009; Niemi \& Näätänen, 1981). The variable-FP effect is often explained in terms of conditional probabilities. Whenever a short FP has elapsed without presentation of the imperative stimulus, the probability of the imperative stimulus at a longer FP is increased (Elithorn \& Lawrence, 1955; Janssen \& Shadlen, 2005; Stuss et al., 2005). When expectancies for the current FP are updated 
accordingly during the course of a trial, the expectancy for the imperative stimulus to occur immediately rises from short to long FPs, leading to better performance for long than for short FPs (see, however, Los \& Agter, 2005; Los \& Heslenfeld, 2005; Los \& Van den Heuvel, 2001; Steinborn, Rolke, Bratzke, \& Ulrich, 2008, for an alternative explanation of the variable-FP effect).

Within the variable-FP paradigm, expectancies can be manipulated by changes in the overall FP distribution. When, for example, one FP appears frequently relative to other FPs, responses are usually faster after the frequently appearing FP (Karlin, 1966; Mowrer, 1940; Nickerson, 1967; Zahn \& Rosenthal, 1966) than after less frequent ones. Another way to manipulate expectancy in the variable-FP paradigm is by informative warning signals. If a warning signal informs participants of which FP will most likely appear in a trial, responses to the imperative stimulus are faster for validly than for invalidly cued FPs (Correa, Lupiáñez, Milliken, \& Tudela, 2004; Correa, Lupiáñez, \& Tudela, 2006b; Coull \& Nobre, 1998).

\section{Specific temporal expectancy}

The vast majority of temporal expectancy research has investigated expectancy for FPs in strict separation from expectancy for events. In order to isolate the effects of temporal expectancy on response performance from any effects of event expectancy, imperative stimuli have always been balanced across FPs (Cardoso-Leite et al., 2009; Ellis \& Jones, 2010; Los \& Schut, 2008; Niemi \& Näätänen, 1981; Pecenka \& Keller, 2009). In such situations, participants can anticipate when an imperative stimulus will appear, but not which stimulus will appear (given that there are several options). We refer to this form of expectancy as general temporal expectancy, to express that temporal expectancy does not include expectancy of a specific event.

Temporal expectancy has also, however, been investigated in a situation in which the expectancy was specific to a certain event. In a variable-FP study by Wagener and Hoffmann (2010b), a neutral warning signal was followed by one of two FPs and one of two target stimuli. Each target symbol was paired frequently with one of the FPs ( $40 \%$ of the trials) and infrequently with the other FP (10\% of all trials). Overall, both target symbols and both FPs appeared equally often. Wagener and Hoffmann (2010b) found that participants adapted to the unequal frequencies of FP-stimulus combinations. Participants responded more quickly and were less error prone for frequent than for infrequent FP-stimulus combinations. This adaptation was explained by associative learning and specific temporal expectancy (Thomaschke, Wagener, Kiesel, \& Hoffmann, 2011). During the course of the experiment, participants learned that each event was associated with a characteristic FP. Based on these associations, participants formed different temporal expectancies for each event. The expectation for each event was scheduled to the FP at which it had frequently appeared in the past (i.e., after its characteristic FP). We refer to this kind of temporal expectancy with the term specific temporal expectancy, to express that a certain FP is expected only relative to a certain stimulus-response event. Note that this definition requires that a certain stimulus be expected only at a specific FP, instead of being equally likely after all possible FPs, as in general temporal expectancy designs.

A central issue in temporal expectancy research is to determine which part of cognitive processing actually benefits from temporal expectancy. In the following section, we review evidence that general temporal expectancy affects motor preparation, improves perception, and facilitates other cognitive capacities. In the experiments presented here, we empirically test whether that evidence also holds true for specific temporal expectancy.

\section{General motor expectancy}

Earlier behavioral studies found evidence for a tight connection between motor preparedness and temporal expectancy. On the one hand, motor-related experimental factors, such as instructed muscle tension or response complexity, modulate expectancy effects from FP distribution on response speed (Sanders, 1980, 1998; Spijkers, 1990). On the other hand, increased temporal expectancy leads to increased response force, a motor-related response parameter (Mattes \& Ulrich, 1997).

These findings are corroborated by recent neuroscientific evidence. Temporal expectancy modulates the activation of spinal cord excitability (Brunia \& Boelhouwer, 1988) and even elicits covert motor activation in the response-relevant muscles (Boulinguez, Jaffard, Granjon, \& Benraiss, 2008). The nature of the effect of temporal expectancy on spinal cord activation seems to strongly depend on the experimental method. In a TMS study with a variableFP design, Van Elswijk, Kleine, Overeem, and Stegeman (2007) found that corticospinal excitability increased with temporal expectancy, and they speculated that expectation synchronizes the spinal motor signal (see also Duque \& Ivry, 2009; Duque, Lew, Mazzocchio, Olivier, \& Ivry, 2010). However, Duclos, Schmied, Burle, Burnet, and Rossi-Durand (2008b) found that in a constant-FP design, expectancy was associated with inhibition in spinal motor neurons (see also Duclos, Burnet, Schmied, \& Rossi-Durand, 2008a; but see also Sinclair \& Hammond, 2009, for an alternative interpretation of these findings). 
Indication for motor inhibition by temporal expectancy has also been shown in the cortical motor system (Boulinguez, Ballanger, Granjon, \& Benraiss, 2009; Sinclair \& Hammond, 2008). In two studies with constant FPs, Tandonnet and colleagues demonstrated that contralateral negativity — an indicator of motor activity — was less variable (Tandonnet, Burle, Vidal, \& Hasbroucq, 2003) and of lower amplitude (Tandonnet, Burle, Vidal, \& Hasbroucq, 2006) in the short-FP condition than in the long-FP condition, suggesting that the cortical motor command is processed with higher efficiency when expectancy is high (see also Fecteau \& Munoz, 2007). However, these results vary depending on the experimental method. For example, Davranche et al. (2007) found, in a TMS/EMG study with a constant-FP paradigm, that increased expectancy leads to increased activation in motor cortical areas, but also leads to inhibition in corticospinal pathways. They concluded that expectancy increases motor preparation, speeding up motor responses, and that expectancy counteracts the cortical motor activation by triggering inhibitory systems in the spinal cord to prevent premature responses (see also Jaffard, Benraiss, Longcamp, Velay, \& Boulinguez, 2007; Jaffard et al., 2008; Tandonnet, Garry, \& Summers, 2010; see Burle, Tandonnet, \& Hasbroucq, 2010, for a recent review of the neuroscience literature on temporal expectancy in the motor system). Although the detailed mechanisms are still under debate, there is a general consensus in the literature that motor processing can be affected by general temporal expectancy.

\section{General nonmotor expectancy}

Other studies have located the effects of temporal expectancy in nonmotor aspects of cognitive processes, such as selection of the response category (Bausenhart, Rolke, Hackley, \& Ulrich, 2006; Fischer, Plessow, \& Kiesel, 2010; Hackley, 2009; Hackley \& Valle-Inclán, 1998, 2003; Los \& Schut, 2008) or perception of target stimuli. With regard to the impact of expectancy on perception, Lange, Rösler, and Röder (2003) showed that EEG markers for perceptual attention were increased at the attended FP, relative to the unattended FP, when participants voluntarily scheduled their auditory attention to one of two FPs, in order to detect loudness-deviant tones (see also Lange \& Heil, 2008; Lange, Krämer, \& Röder, 2006). Accordingly, Bausenhart, Rolke, and Ulrich (2007) found, in a pitch discrimination paradigm with constant FPs, that shorter tones (i.e., less perceptual input) are sufficient for participants to discriminate pitch when the fixed FP is short (i.e., when temporal expectancy is high; see above).

Evidence for temporal expectancy effects has also been provided for tactile (Lange \& Röder, 2006) and visual stimuli (Correa, Lupiáñez, \& Tudela, 2005). In particular, temporal preparation seems to increase the temporal resolution of the visual system. Temporal order judgments are facilitated when the stimuli appear at an expected point in time (Bausenhart, Rolke, \& Ulrich, 2008; Correa, Sanabria, Spence, Tudela, \& Lupiáñez, 2006c), and intervals tend to be perceived as longer when they appear at expected times (Grondin \& Rammsayer, 2003). A further account of perceptual temporal expectancy claims that temporal expectancy speeds up the detection of stimulus onset (early-onset hypothesis; see Rolke \& Hofmann, 2007; Seibold, Bausenhart, Rolke, \& Ulrich, 2011a; Seibold, Fiedler, \& Rolke, 2011b; Seifried, Ulrich, Bausenhart, Rolke, \& Osman, 2010).

With regard to a potential perceptual locus of specific temporal expectancy, a related study by Kingstone (1992) is of interest. He investigated the interaction between general event expectancy and general temporal expectancy. In his study, the target letters $\mathrm{A}$ and $\mathrm{V}$ were displayed either upright or upside down. The task was to identify the orientation (upright or upside down) of the letters. He manipulated event expectancy and temporal expectancy by dual cues. One part of the cue predicted the FP, and the other part of the cue predicted stimulus form (i.e., letter identity). Thus, on each trial, participants formed expectancies concerning the FP and the stimulus form. The crucial difference to the previously described phenomenon of specific temporal expectancy was that, in Kingstone's study, the probabilities of FP and event were not conditional upon each other. The event cue increased the probability of the cued form at the cued and at the noncued time to the same degree. Likewise, the time cue increased the probability of the cued FP for the cued form and for the noncued form to the same degree. Participants adapted to the informative cues, as evidenced by the fastest responses occurring when stimulus form and time were both cued validly. Importantly, only stimulus form was cued by the event cue, while responses were mapped to stimulus orientation, and thus were not cued. This means that the cued expectancy was of a perceptual nature. Hence, general event expectancy and general temporal expectancy can, when they coincide, simultaneously facilitate perceptual processing. This suggests that specific temporal expectancy-defined as expectancy for a combination of event and time-might also affect perceptual processing.

In conclusion, there is accumulating evidence that general temporal expectancy affects many aspects of cognitive processing, from stimulus perception to response execution. It is not known, however, which processes are affected by specific temporal expectancies. Previous studies were not able to dissociate between specific temporal expectancy effects on different cognitive processes (Haering \& Kiesel, in press; Wagener \& Hoffmann, 2010a, 2010b). In these studies, fast responses for frequent $\mathrm{FP}-$ stimulus 
combinations could have been due to a facilitation of perception, response execution, or any neural process between the two.

The present study aimed to disentangle the effects of specific temporal expectancy on stimulus perception from effects on more response-related processes.

\section{General method}

\section{Overview}

In a speeded binary choice task with two variable FPs, we used four stimuli that differed along two binary dimensions: orientation (vertical vs. horizontal) and form (diamond vs. oval). Stimulus frequency was unequally distributed over two different FPs, meaning that each stimulus appeared frequently with one FP and infrequently with the other FP. In this way, one stimulus dimension could be equally distributed over FPs, while at the same time the other stimulus dimension could be correlated with the FPs. For instance, form could be correlated with FP (e.g., diamonds often appeared after a short FP, and ovals often appeared after a long FP), while orientation could be equally distributed over both FPs.

In Experiments 1 and 3, the responses were mapped to stimuli according to the same stimulus dimension that was correlated with the FPs; therefore, the response was also correlated with FPs. In Experiments 2 and 4, the responses were mapped to stimuli according to a dimension that was not correlated with the FPs. As a result, stimuli were FPcorrelated with regard to the other dimension, but responses were not (see Table 1).

In Experiment 1, participants responded to either stimulus form (Exp. 1A) or stimulus orientation (Exp. 1B), and either stimulus form (Exp. 1A) or stimulus orientation (Exp. 1B) was correlated with FPs. Thus, FPs and responses were both correlated with either stimulus form or with stimulus orientation. This experiment replicated previous studies on the specific temporal adaptation

Table 1 Stimulus-response mappings and correlations with foreperiod length in Experiments 1-4

\begin{tabular}{lll}
\hline Experiment & Response was mapped to: & FP was correlated with: \\
\hline 1A & Form & Form \\
1B & Orientation & Orientation \\
2A & Form & Form $\times$ Orientation \\
2B & Orientation & Form $\times$ Orientation \\
3 & Form $\times$ Orientation & Form $\times$ Orientation \\
$4 \mathrm{~A}$ & Form & Orientation \\
$4 \mathrm{~B}$ & Orientation & Form \\
\hline
\end{tabular}

effect and was meant to validate the new stimulus material. In Experiment 2, FPs were correlated with a combination of form and orientation, while responses were mapped according to either form (Exp. 2A) or orientation (Exp. 2B). Thus, responses were not correlated with FP. In Experiment 3, FPs were again correlated with a combination of form and orientation, but responses were also mapped to the combinations of form and orientation, so that now responses were correlated to FPs. In Experiment 4, we correlated the FP with one stimulus dimension (either form or orientation) and mapped the responses to the other stimulus dimension (orientation or form).

\section{Participants}

Participants were either students, who received course credits, or inhabitants of the city of Würzburg, who received $€ 5$ for participation. All participants had normal or corrected-tonormal vision and gave informed consent before participation. Participants were treated according to the ethical standards of the American Psychological Association.

Apparatus and stimuli

Stimulus presentation and collection of responses were performed by an IBM-compatible computer with a 17-in. VGA display controlled by E-Prime (Schneider, Eschman, \& Zuccolotto, 2002). Participants responded with the right hand on two adjacent buttons on a serial response box (Psychology Software Tools), which was centrally aligned in front of the computer screen. The stimuli were a black diamond or a black oval, presented either vertically or horizontally (see Fig. 1) against a white background, at a viewing distance of $50 \mathrm{~cm}$. The size of the stimuli was $2 \times$ $1 \mathrm{~cm}$. The fixation cross was the "+" symbol (Arial typeface, $1.3 \times 1.3 \mathrm{~cm}$ ). All stimuli were presented centrally on the screen.

\section{Procedure}

Participants had to respond in each trial to one of four stimuli. They were instructed to respond to two of the
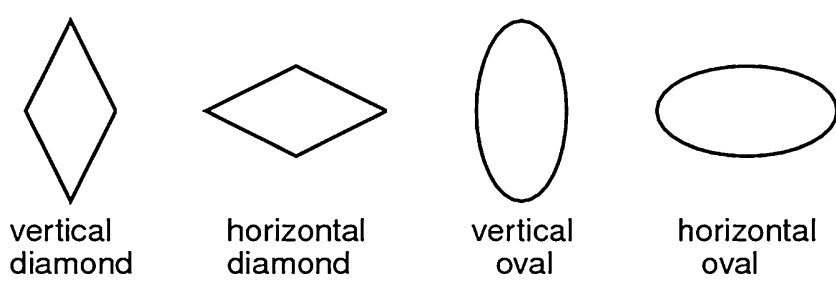

Fig. 1 Illustration of the experimental stimuli. The diamond was a regular rhombus with the length of one diagonal half the length of the other diagonal. The oval was a regular ellipse with a conjugate diameter of half the length of the traverse diameter 
stimuli with the left response button and to the other two stimuli with the right response button. They were not informed that FPs had different lengths, or that these FP lengths were correlated with stimuli.

See Figure 2 for a diagram of the overall procedure. Each trial started with the presentation of the fixation cross for a variable FP of either 600 or 1,600 ms. The target stimulus was then presented. The order of stimuli was randomized, and each stimulus occurred with equal probability. Each stimulus was preceded by one of the two FPs in $80 \%$ of its occurrences (frequent combination of symbol and FP), and was preceded by the other FP in the other $20 \%$ (infrequent combination of symbol and FP). Participants were not informed about this regularity. The mapping of the responses to the symbols was counterbalanced between participants. The time window for responses was $1,100 \mathrm{~ms}$, starting from the presentation of the target stimulus. When no response was given during this time window, the words zu spät! (German for "too late!") were displayed for $700 \mathrm{~ms}$. When participants pressed the wrong key, the words falsche Taste! (German for "wrong key!") were displayed for $700 \mathrm{~ms}$. After correct and timely responses, no explicit feedback was given. After trials with incorrect or late responses, the next trial started $500 \mathrm{~ms}$ after offset of the error message. After trials with timely and correct responses, the next trial started 1,200 ms after responding.

Participants completed four blocks of 300 trials each. They performed 60 trials for each of the infrequent combinations of stimulus and FP, and 240 trials for each of the frequent combinations of stimulus and FP. Between the blocks, participants could take a short break of $1 \mathrm{~min}$. The experiment lasted approximately $1 \mathrm{~h}$.

Data processing and analyses

Data from the first experimental block and from the first 4 trials from each remaining block were excluded from analyses. For each combination of block (2-4), FP (600 vs. 1,600 ms), and frequency of FP-symbol combination (frequent vs. infrequent), mean error rates and RTs were calculated separately for each participant. We included the factor Block in the analysis to assess whether the effect of the frequency of an FP-symbol combination evolved during the course of the experiment.

Trials with errors were removed from the RT analyses. For each factor combination and participant, RTs with a deviation of more than $2.5 S D$ from the participant's mean RT for that combination were removed before the RT analysis (Bush, Hess, \& Wolford, 1993). Three-factor repeated measures ANOVAs with the factors Block (2-4), FP (600 vs. 1,600 ms), and Frequency of FP-Symbol Combination (frequent vs. infrequent) were conducted separately for error rates and RTs. Significance for any factor or factor combination did not differ between subexperiments - that is, between whether orientation or form was correlated to response or FP. Consequently, we collapsed the data for all subexperiments.

\section{Experiment 1}

The purposes of Experiments 1A and 1B were to replicate Wagener and Hoffmann's (2010b) results with a new set of stimuli and to serve as a baseline for further experiments. In Experiment 1A, participants responded to the form of the stimuli, and FPs were correlated with form. In Experiment 1B, participants responded according to the orientation of the stimuli, and FPs were correlated with orientation. We expected a specific temporal expectancy effect for both subexperiments. We also expected the effect to increase during the course of the experiment, since we assumed the adaptation process to be due to associative learning.
Fig. 2 Trial structures of a longFP and a short-FP trial. The left figure illustrates a trial in which the FP was long, the symbol was a horizontal oval, and the response was correct. The right figure illustrates a trial in which the FP was short, the symbol was a vertical diamond, and the response was correct
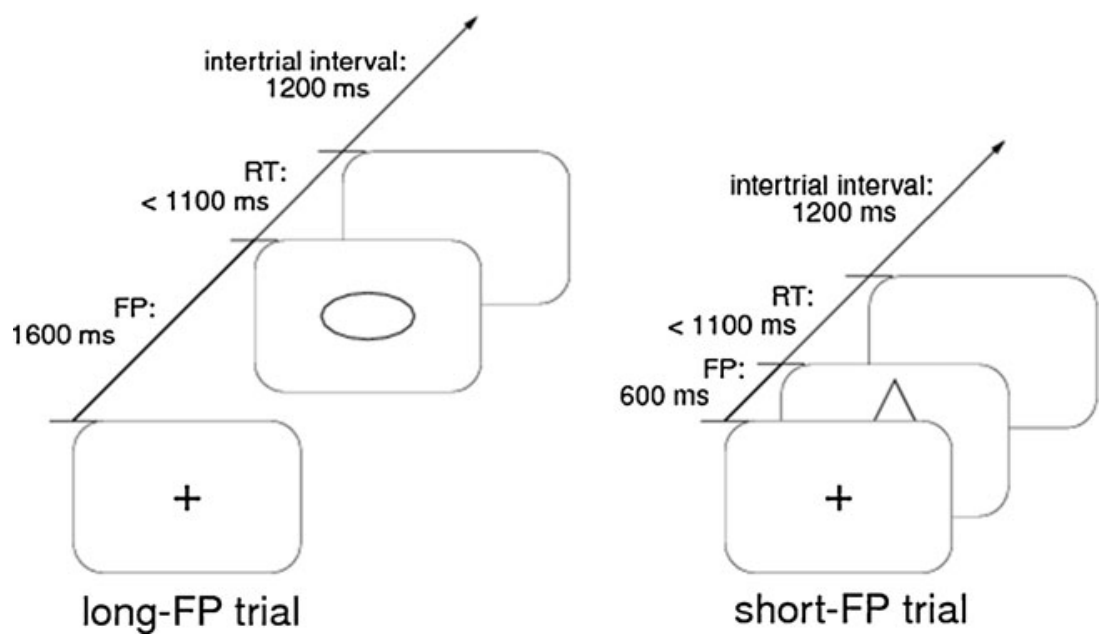
Method

\section{Participants}

A group of 32 participants took part in the experiment, 16 in Experiment $1 \mathrm{~A}$ and 16 in Experiment 1B. The participants' ages ranged from 18 to 47 years, $M=23.16$, $S D=5.3$. Twenty of the participants were female, and 12 were male. For 1 participant in Experiment 1A, no data were saved due to technical problems.

\section{Procedure}

In Experiment 1A, participants were instructed to respond as quickly and correctly as possible with one response button to the diamond and with the other response button to the oval. They were not informed that the FPs were of any relevance to the experiment or that the FPs differed in length. One form (diamond or oval) occurred frequently after one of the two possible FPs (600 or 1,600 ms), while the other form appeared frequently after the other FP (see Table 2). Overall, both FPs and forms appeared with the same frequencies.

In Experiment 1B, participants were instructed to respond with one response button to vertical symbols and with the other response button to horizontal symbols. They were not informed that the FPs differed in length. One orientation (vertical or horizontal) occurred frequently after one of the two possible FPs ( 600 or $1,600 \mathrm{~ms}$ ), while the other orientation appeared frequently after the other FP. Overall, both FPs and orientations appeared with the same frequencies. The mappings of forms and orientations to FPs and responses, respectively, were counterbalanced across participants.

\section{Results}

Mean RTs and error rates as a function of block and frequency of $\mathrm{FP}$-symbol combinations are depicted in Figure 3. With regard to RTs, the three main effects were significant. Responses were faster after long than after short FPs, $F(1,30)=18.65, p<.001, \eta_{p}^{2}=.383$, and responses to frequent $\mathrm{FP}$-symbol combinations were faster than responses after infrequent combinations, $F(1,30)=$ 52.278, $p<.001, \eta_{p}^{2}=.635$ (see also Table 3). The factor Block showed a significant linear trend, $F(1,30)=10.40, p=$ $.003, \eta_{p}^{2}=.257$, with decreasing RTs from Block 2 to Block 4. The only significant interaction for RTs was between block and frequency, $F(2,60)=3.771, p=.029, \eta_{p}^{2}=.112$. The effect of frequency was larger in Blocks 3 and 4 (see Fig. 3), but the linear trend was not significant, $F(1,39)=1.105, p=$ $.302, \eta_{p}^{2}=.036$.

With regard to error rates, the main effect of frequency was significant, $F(1,30)=13.13, p=.001, \eta_{p}^{2}=.304$, with fewer errors for frequent than for infrequent FP-symbol combinations. No other main effect or interaction was significant.
Table 2 Examples for correlations of responses and typical FPs with symbols in Experiments 1-4
The mapping of responses to symbols was fixed for each participant throughout the experiment. That FPs were correlated with a symbol, which means that each symbol was preceded by that FP in $80 \%$ of its occurrences (frequent combination of symbol and FP), while the symbol was preceded in only $20 \%$ of its occurrences by the alternative FP (infrequent combination of symbol and FP)

\begin{tabular}{|c|c|c|c|c|}
\hline & Vertical Diamond & Horizontal Diamond & Vertical Oval & Horizontal Oval \\
\hline \multicolumn{5}{|l|}{ Experiment $1 \mathrm{~A}$} \\
\hline Response & left & left & right & right \\
\hline Frequent FP & short & short & long & long \\
\hline \multicolumn{5}{|l|}{ Experiment 1B } \\
\hline Response & left & right & left & right \\
\hline Frequent FP & short & long & short & long \\
\hline \multicolumn{5}{|l|}{ Experiment $2 \mathrm{~A}$} \\
\hline Response & left & left & right & right \\
\hline Frequent FP & short & long & long & short \\
\hline \multicolumn{5}{|l|}{ Experiment 2B } \\
\hline Response & left & right & left & right \\
\hline Frequent FP & short & long & long & short \\
\hline \multicolumn{5}{|l|}{ Experiment 3} \\
\hline Response & left & right & right & left \\
\hline Frequent FP & short & long & long & short \\
\hline \multicolumn{5}{|l|}{ Experiment $4 \mathrm{~A}$} \\
\hline Response & left & left & right & right \\
\hline Frequent FP & short & long & short & long \\
\hline \multicolumn{5}{|l|}{ Experiment 4B } \\
\hline Response & left & right & left & right \\
\hline Frequent FP & short & short & long & long \\
\hline
\end{tabular}


Fig. 3 Mean error rates and RTs for frequent and infrequent FP-symbol combinations as a function of block in Experiment 1. Error bars represent $\pm 1 S E$. Asterisks denote significance at the .05 level, and double asterisks denote significance at the .001 level

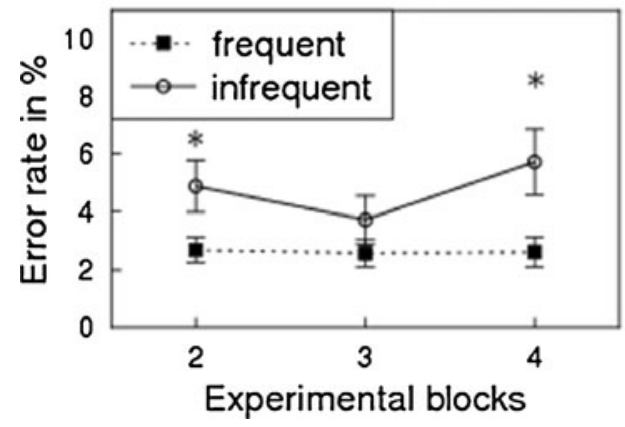

\section{Discussion}

Participants responded more quickly and more accurately for frequent $\mathrm{FP}-$ symbol combinations than for infrequent ones. This means that participants adapted to the correlation between form and FP in Experiment 1A, and to the correlation between orientation and FP in Experiment 1B. This shows that specific temporal expectancy takes place in choice reactions when the task-relevant stimulus dimension is form (as in Wagener \& Hoffmann, 2010b, and Exp. 1A), or orientation (as in Exp. 1B). The interaction between block and frequency confirmed that temporal expectancy was acquired over the course of the experiment, supporting an associative learning account of the phenomenon. It is, however, not possible to conclude whether participants adapted to correlations between responses and FPs or to correlations between stimuli and FPs, because stimuli and responses were confounded.

\section{Experiment 2}

The purpose of Experiment 2 was to test whether a specific temporal expectancy effect can be observed when the stimuli, but not the responses, correlate with FPs. To this end, a combination of stimulus features was correlated with FPs, while responses were mapped onto a single feature dimension (form in Exp. 2A and orientation in Exp. 2B), and consequently were balanced across FPs. Because each response appeared at the same frequency after the short FP and after the long FP, response-specific temporal expectancy was not possible in this experiment. A specific temporal expectancy effect could only be due to a temporally specific expectancy for the individual symbols at their typical FPs. Thus, the occurrence of a specific temporal expectancy effect would support a perceptual facilitation account. Absence of a specific temporal adaptation effect would suggest that the effect in Experiment 1 was due to expectancy for response-related processes.

Method

\section{Participants}

There were 16 participants in each of Experiments 2A and 2B. The participants' ages ranged from 19 to 45 years, $M=25.06, S D=4.9$. Twenty-two were female, and 10 were male.
Table 3 Mean error rates and response times for frequent and infrequent combinations of a symbol and FP at the short and the long FPs

\begin{tabular}{|c|c|c|c|c|c|c|c|c|}
\hline \multirow[b]{3}{*}{ Experiment } & \multicolumn{4}{|l|}{$600 \mathrm{~ms}$} & \multicolumn{4}{|c|}{$1,600 \mathrm{~ms}$} \\
\hline & \multicolumn{2}{|c|}{ Frequent } & \multicolumn{2}{|c|}{ Infrequent } & \multicolumn{2}{|c|}{ Frequent } & \multicolumn{2}{|c|}{ Infrequent } \\
\hline & $M$ & $S D$ & $M$ & $S D$ & $M$ & $S D$ & $M$ & $S D$ \\
\hline \multicolumn{9}{|c|}{ Response Times } \\
\hline 1 & 400 & 50 & 413 & 50 & 384 & 48 & 405 & 48 \\
\hline 2 & 427 & 35 & 432 & 41 & 411 & 39 & 407 & 49 \\
\hline 3 & 600 & 84 & 632 & 92 & 625 & 87 & 621 & 97 \\
\hline 4 & 419 & 53 & 422 & 51 & 415 & 51 & 413 & 54 \\
\hline \multicolumn{9}{|l|}{ Error Rates } \\
\hline 1 & 3.65 & 3.4 & 5.26 & 3.84 & 2.34 & 1.9 & 4.83 & 5.7 \\
\hline 2 & 3.99 & 7.6 & 4.42 & 9.75 & 3.53 & 7.2 & 4.20 & 7.9 \\
\hline 3 & 6.29 & 4.7 & 8.29 & 8.0 & 7.24 & 6.7 & 7.94 & 7.2 \\
\hline 4 & 3.65 & 4.0 & 2.93 & 4.2 & 3.10 & 3.4 & 2.29 & 3.7 \\
\hline
\end{tabular}




\section{Procedure}

In Experiment 2A, participants were instructed to respond with one response button to diamonds and with the other to ovals. In Experiment 2B, they were instructed to respond with one response button to vertical symbols and with the other to horizontal symbols. The mappings of the respective features to the responses (left, right) were counterbalanced across participants.

In both subexperiments, the vertical oval and the horizontal diamond appeared frequently after one of the FPs, while the horizontal oval and the vertical diamond appeared frequently after the other FP. The combinations of FP with the set of stimuli were counterbalanced across participants. Neither single stimulus dimension was correlated with FPs; that is, the diamond and the oval appeared equally often after the short and after the long FP. Likewise, the stimuli were equally often vertically or horizontally oriented after the short FP and after the long FP.

\section{Results}

Mean RTs and error rates are shown in Figure 4. With regard to RTs, the main effects for FP and block were significant. Responses were faster after long than after short FPs, $F(1,31)=27.15, p<.001, \eta_{p}^{2}=.467$, and the effect of block, $F(2,62)=5.485, p=.006, \eta_{p}^{2}=.150$, was again due to a linear trend toward faster responses in later blocks, $F(1,31)=7.299, p=.011, \eta_{p}^{2}=.191$. The main effect of the frequency of FP-symbol combinations was not significant, $F(1,31)=0.134, p=.717, \eta_{p}^{2}=.004$, nor was any interaction. With regard to error rates, no main effect or interaction was significant (all $p \mathrm{~s}>.2$ ).

\section{Discussion}

Neither error rates nor RTs revealed any evidence of a specific temporal expectancy effect. This suggests that behavioral adaptation to FP-stimulus correlations requires that responses also be correlated with FPs. This, in turn, indicates that specific temporal expectancy is related to responses, rather than to stimuli.
However, one might object to the idea that specific temporal expectancy could indeed affect perception, but only the perceptual processing of simple stimulus features. Despite the fact that general temporal expectancy effects were observed for basic visual feature processing (Bueti, Bahrami, Walsh, \& Rees, 2010; Rolke \& Hofmann, 2007; Seifried et al., 2010) and for complex visual features (Correa, Cappucci, Nobre, \& Lupiáñez, 2010a; Correa, Lupiáñez, Madrid, \& Tudela, 2006a; Hackley, Schankin, Wohlschlaeger, \& Wascher, 2007; Rolke, 2008), specific temporal expectancy might be restricted to perceptual processing of simple stimulus features.

In Experiment 2, complex feature combinations, instead of simple features, were correlated with FPs. If one assumes that only the discrimination of simple features benefits from specific temporal expectancy, the absence of an effect in Experiment 2 would be consistent with a perceptual account of specific temporal expectancy. In order to test this alternative explanation, we conducted two further experiments. In Experiment 3, complex stimulus feature combinations were again correlated with FPs, but responses were also correlated with FPs. If specific temporal expectancy only facilitates perception of simple stimulus features, we would not expect an effect of frequency of FPsymbol combinations. On the other hand, if specific temporal expectancy facilitates response preparation, we would expect to see an effect. In Experiment 4, one simple feature dimension (form or orientation) was correlated with FPs, whereas responses were mapped onto the other feature dimension, and hence were uncorrelated with FPs. If specific temporal expectancy facilitates responses, we would not expect to find a specific temporal expectancy effect in Experiment 4. However, if specific temporal expectancy facilitates perceptual processing of simple stimulus features, we would expect to find an adaptation effect.

\section{Experiment 3}

The purpose of Experiment 3 was to discriminate between two opposing interpretations of Experiment 2-namely that specific temporal expectancy either facilitates perception of
Fig. 4 Mean error rates and RTs for frequent and infrequent FP-symbol combinations as a function of block in Experiment 2. Error bars represent $\pm 1 S E$. The means are displayed separately for each analyzed block of the experiment
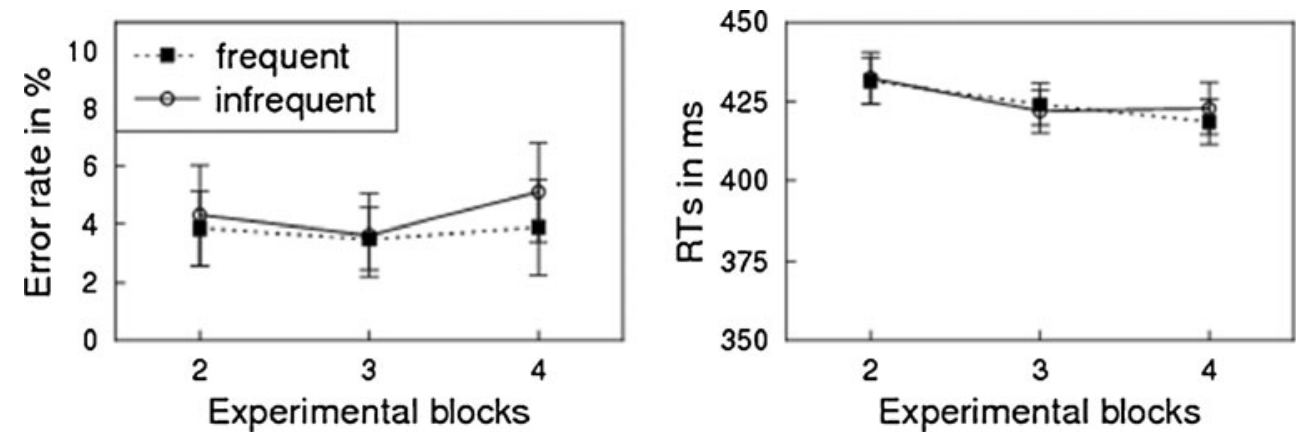
simple stimulus features or facilitates nonperceptual, response-related processes. FPs were correlated with complex stimulus features (as in Exp. 2), and responses were mapped to stimuli according to these complex stimulus features. This had the effect that responses were also correlated with FPs. If specific temporal expectancy facilitates perception of simple stimulus features, we should not find an expectancy effect, because single feature dimensions were balanced across FPs, and only complex feature combinations were correlated with FPs. If specific temporal expectancy is, on the other hand, nonperceptual and response related, we should find a specific temporal expectancy effect, because responses were correlated with FPs. Note, however, that the task was more difficult than in the previous experiments. Participants had to select responses according to arbitrary stimulus classes (e.g., a horizontal oval and a vertical diamond were mapped to one response button), while in previous experiments responses were selected according to a single stimulus dimension (e.g., only diamonds were mapped to the right response button).

\section{Method}

\section{Participants}

A group of 20 participants, 16 females and 4 males, took part in the experiment. Their ages ranged from 19 to 40 years, $M=23.80, S D=5.3$. For 1 participant, no data were saved due to technical problems.

\section{Procedure}

The vertical oval and the horizontal diamond appeared frequently after one FP, while the horizontal oval and the vertical diamond appeared frequently after the other FP. Participants were instructed to respond with one button to the vertical oval or the horizontal diamond, and to respond with the other button to the horizontal oval or the vertical diamond. The mappings between the stimulus set and responses were counterbalanced across participants. Allocation of the stimulus set to FP frequencies was also counterbalanced across participants.

Form and orientation were balanced over FPs, in the sense that the diamond as well as the oval, and vertical as well as horizontal orientations, appeared equally often after the short and the long FP.

\section{Results}

Mean RTs and error rates as a function of block and of frequent versus infrequent $\mathrm{FP}$-symbol combinations are shown in Figure 5. Note that the RTs and error rates are generally higher than in the previous experiments. This was due to the more difficult task demands to respond to feature combinations (see above).

With regard to RTs, the only significant main effect was of frequency, $F(1,18)=8.908, p=.008, \eta_{p}^{2}=.331$, with responses to frequent $\mathrm{FP}$-symbol combinations being faster than responses to infrequent FP-symbol combinations. The factor Block was marginally significant, $F(2,36)=2.979$, $p=.064, \eta_{p}^{2}=142$, with a significant linear trend toward faster responses for later blocks, $F(1,18)=5.865, p=.026$, $\eta_{p}^{2}=.246$. No interaction was significant (all $p>.23$ ).

With regard to error rates, the only significant main effect was of block, $F(2,36)=7.156, p=.002, \eta_{p}^{2}=.284$. This effect could be further qualified as a linear trend toward fewer errors for later blocks, $F(1,18)=12.495, p=.002$, $\eta_{p}^{2}=.410$. The effect of frequency was not significant overall, $F(1,18)=0.134, p=.718, \eta_{p}^{2}=.007$. We also analyzed the effect of frequency separately for Blocks 2-4. Error rates were significantly higher for infrequent than for frequent $\mathrm{FP}$-stimulus combinations in Block 2, $t(18)=$ 2.366, $p=.029$, but not in Blocks 3 and $4(p s>.49)$. No interaction was significant (all $p \mathrm{~s}>.29$ ).

\section{Discussion}

We found a specific temporal expectancy effect in RTs and, in the first analyzed block, also in error rates. The results further support our interpretation of Experiment 2 that specific temporal expectancy is nonperceptual. In Experiment 2, FPs correlated with perceptual feature combinations, but not with responses, and we found no adaptation effect. In Experiment 3, FPs correlated with perceptual feature combinations and responses, and we did find an adaptation effect. The only difference between Experiments 2 and 3 was that responses were correlated with FPs. We concluded that specific temporal expectancy facilitates response preparation. The alternative hypothesis, that specific temporal expectancy is perceptual but cannot be formed for feature combinations (only for simple features), is not confirmed. To confirm this hypothesis, no specific temporal expectancy effect should emerge in either of the following experiments, because simple features were not correlated with FP in these experiments.

\section{Experiment 4}

The purpose of Experiment 4 was to further test whether specific temporal expectancy facilitates the perception of simple stimulus features or facilitates response preparation. In Experiment 4, FPs were correlated with one stimulus dimension (form in Exp. 4B, and orientation in Exp. 4A), but responses were mapped to stimulus features, according to the other stimulus dimension (form in Exp. 4A, and 
Fig. 5 Mean error rates and RTs for frequent and infrequent $\mathrm{FP}-$ symbol combinations as a function of block in Experiment 3. Error bars represent $\pm 1 S E$. The means are displayed separately for each analyzed block of the experiment, and the asterisk denotes significance at the .05 level

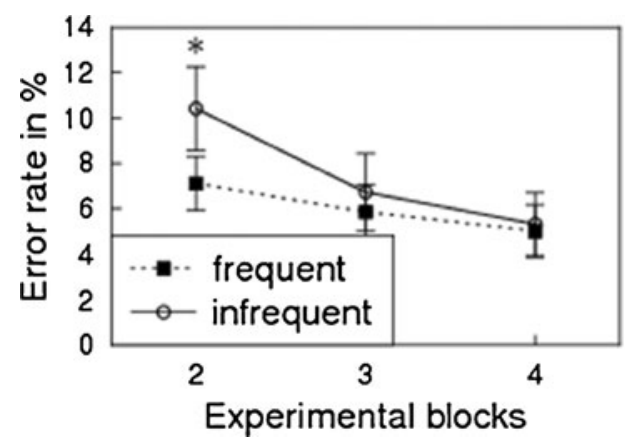

orientation in Exp. 4B). If specific temporal expectancy facilitates perception of simple features, we should find a clear specific temporal expectancy effect, because a simple feature dimension is correlated with FPs. If specific temporal expectancy facilitates response-related processes, no specific temporal expectancy effect should occur, because responses were not correlated with FPs.

Method

\section{Participants}

There were 16 participants in each of Experiments 4A and 4B. The participants' ages ranged from 18 to 60 years, $M=24.66$, $S D=7.5$. Twenty-five were female, and 7 were male.

\section{Procedure}

In Experiment 4A, vertically oriented stimuli appeared frequently after one FP, while horizontally oriented stimuli appeared frequently after the other FP. Participants were instructed to respond with one response button to the diamond and with the other to the oval. The correlation between FPs and orientations, as well as the mapping of forms to responses, were counterbalanced across participants.

In Experiment 4B, diamonds were frequent after one FP, while ovals were frequent after the other FP. Participants were instructed to respond with one response button to vertical symbols and with the other to horizontal symbols. The correlation between FPs and form, as well as the mapping of forms to responses, was counterbalanced across participants.

\section{Results}

Mean RTs and error rates as a function of block and of frequent versus infrequent $\mathrm{FP}-$ symbol combinations are shown in Figure 6. With regard to RTs, the main effects of FP and block were significant. Responses were faster after long than after short FPs, $F(1,31)=4.428$, $p=.044, \eta_{p}^{2}=.125$, and the effect of block, $F(2,62)=$ $5.175, p=.008, \eta_{p}^{2}=.143$, was again due to a linear trend toward faster responses in later blocks, $F(1,31)=$
$8.880, p=.006, \eta_{p}^{2}=.223$. The main effect of frequency was not significant, $F(1,31)=0.035, p=.852$, $\eta_{p}^{2}=.001$. No interaction was significant (all $p \mathrm{~s}>.39$ ).

With regard to error rates, no main effect or interaction was significant (all $p \mathrm{~s}>.079$ ). Most importantly, the main effect of frequency was not significant, $F(1,31)=3.302$, $p=.079, \eta_{p}^{2}=.096$, and was actually numerically reversed, with more errors for frequent than for infrequent FP-symbol combinations.

\section{Discussion}

We did not observe a specific temporal expectancy effect in either RTs or error rates. The absence of an effect when only a simple feature dimension was correlated with FPs clearly speaks against a perceptual account of specific temporal expectancy. The results support the notion that specific temporal expectancy is based on nonperceptual, responserelated processes.

\section{Summary and general discussion}

We conducted four experiments in order to determine whether specific temporal expectancy affects perceptual processing or is associated with nonperceptual, response-related processing. The experiments provided consistent evidence for a nonperceptual locus of specific temporal expectancy. Experiment 1 replicated earlier findings that demonstrated that participants adapt to frequencies of FP-event combinations when one stimulus feature and the responses correlate with the FPs. In Experiment 2, participants did not adapt to correlations between stimulus features and FPs when responses did not correlate with the FPs. In Experiment 3, a specific temporal expectancy effect was found when stimulus feature combinations as well as responses correlated with the FPs. Lastly, in Experiment 4, participants did not adapt to a correlation of simple stimulus features with FPs when responses did not correlate with the FPs. In sum, we found evidence for specific temporal expectancy only when responses were correlated with FPs (irrespective of whether the responses were assigned to simple stimulus 
Fig. 6 Mean error rates and RTs for frequent and infrequent $\mathrm{FP}-$ symbol combinations as a function of block in Experiment 4. Error bars represent $\pm 1 S E$. The means are displayed separately for each analyzed block of the experiment
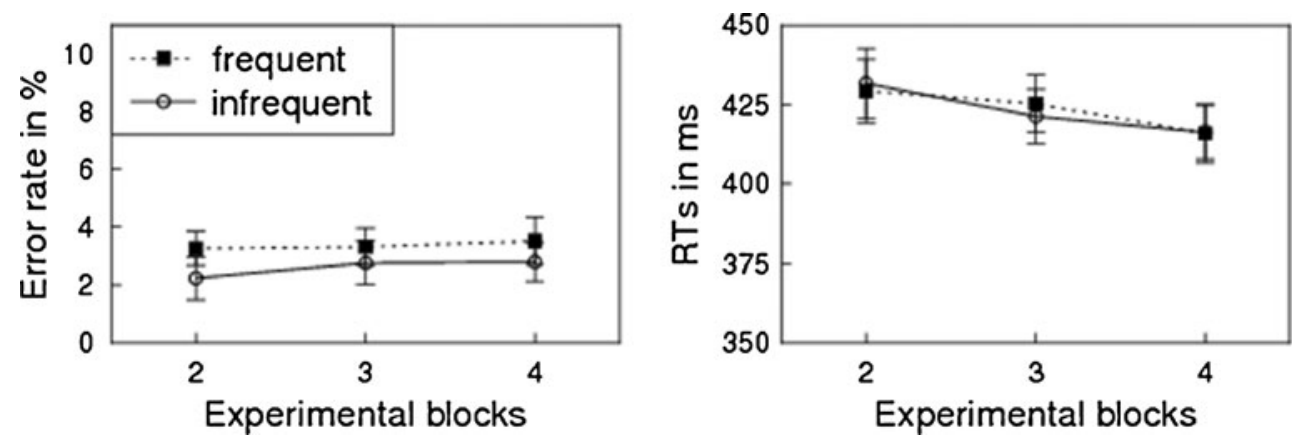

features, as in Exp. 1, or to a combination of stimulus features, as in Exp. 3). When just the stimuli were correlated with FPs, but not the responses, no specific temporal expectancy effect was observed (Exps. 2 and 4).

We conclude from these results that specific temporal expectancy is mainly expectancy for nonperceptual cognitive events. While perception can benefit from general temporal expectancy (Correa et al., 2005; Lange, 2009; Rimmele, Jolsvai, \& Sussman, 2010; Rolke, 2008), in the sense of facilitated perceptual processing at a certain point in time, this benefit cannot be specific for a certain stimulus. This finding is particularly remarkable in relation to Kingstone's (1992) study, which showed that general event expectancy and general temporal expectancy can simultaneously facilitate perceptual processing. This suggests that the coincidence of unconditional event and unconditional FP expectancies relies on functionally different cognitive mechanisms rather than on specific temporal expectancies (i.e., expectancies for combinations of event and FP).

This conclusion is further supported by the lack of interaction between the variable-FP effect and the specific temporal expectancy effect in any of the experiments. When the short FP has elapsed in a trial, the probability that a stimulus will appears after the long FP increases from .5 to 1, typically leading to overall faster RTs after long than after short FPs - that is, the variable-FP effect (see the introduction). We found a variable-FP effect in each experiment except Experiment 3. The effect did not, however, interact with the specific temporal expectancy effect. In addition, the presence and size of the variable-FP effect also did not systematically depend on the presence of specific temporal expectancy in the experiment. In Experiment 1, with specific temporal expectancy, we found that the variable-FP effect was of medium size $\left(\eta_{p}^{2}=.383\right)$. In experiments without specific temporal expectancy, the variable-FP effect was small $\left(\eta_{p}^{2}=.125\right.$, Exp. 4) or large $\left(\eta_{p}^{2}=.467\right.$, Exp. 2). This suggests that there is one kind of temporal expectancy based on elapsed time within a trial, and another, specific temporal expectancy based on prior associative pairings of stimulus-response episodes and FPs.

Although the present experiments have shown that specific temporal expectancy affects nonperceptual process- ing, our results do not allow for a more detailed differentiation between the affected cognitive processes. These results would be compatible with a motor locus of specific temporal expectancy. It might be that specific temporal expectancy for a certain symbol at a certain FP increases the spinal cord excitability for the response assigned to this stimulus, similar to the results shown by Brunia and Boelhouwer (1988) for general temporal expectancy (see the introduction). On the other hand, some authors have demonstrated that temporal expectancy affects response selection stages. Broadbent and Gregory (1965), for example, showed that the length of an FP had a larger effect on RTs when the mapping of stimuli to responses was congruent than when it was incongruent, which suggests that general expectancy is associated with response selection. A response selection locus of specific temporal expectancy would also be compatible with the present findings. Participants might have scheduled their expectancy for selecting one of two responses to the FP at which the response was frequently required.

The discussion of specific temporal expectancy is, in the present study, entirely focused on frequency-induced expectancy in variable-FP designs. This is because the effect has previously only been shown in this kind of paradigm. However, specific temporal expectancy may also emerge in other temporal expectancy paradigms. As mentioned in the introduction, participants respond more quickly when the current FP was validly predicted by a preceding cue than when the cue had predicted another FP. We propose that an analogous cuing effect would result for the cuing of combinations of FP and target. Such an effect would demonstrate specific temporal expectancy based on cues. We also expect that in a constant-FP paradigm, participants would also specifically temporally expect an FP-event combination when one stimulus-response event primarily appeared in blocks with one certain FP and another event primarily occurred in blocks with another FP. Furthermore, we propose that specific temporal expectancy plays an important role in the perception of rhythms, because rhythmical sequences are, in everyday life, usually coupled with a characteristic sequence of events (e.g., musical rhythms). The temporal expectancy induced by a rhythmical sequence has been shown to interact with the 
variable-FP effect for randomly distributed sequence-final FPs (Ellis \& Jones, 2010; Sanabria, Capizzi, \& Correa, 2011). However, whether rhythm also interacts with specific temporal expectancy in a sequence-final FP has not been tested.

To conclude, the present results have revealed that specific temporal expectancy affects postperceptual processing stages. However, more research will be required in order to further differentiate between motor activation and response selection as potential loci of specific temporal expectancy.

Author Note This research was supported by Grant HO 1301/13-1 awarded by the Deutsche Forschungsgemeinschaft.

\section{References}

Awramoff, D. (1903). Arbeit und Rhythmus: Der Einfluss des Rhythmus auf die Quantität und Qualität geistiger und körperlicher Arbeit, mit besonderer Berücksichtigung des rhythmischen Schreibens. Philosophische Studien, 18, 515-562.

Bausenhart, K. M., Rolke, B., Hackley, S. A., \& Ulrich, R. (2006). The locus of temporal preparation effects: Evidence from the psychological refractory period paradigm. Psychonomic Bulletin \& Review, 13, 536-542. doi:10.3758/BF03193882

Bausenhart, K. M., Rolke, B., \& Ulrich, R. (2007). Knowing when to hear aids what to hear. Quarterly Journal of Experimental Psychology, 60, 1610-1615. doi:10.1080/17470210701536419

Bausenhart, K. M., Rolke, B., \& Ulrich, R. (2008). Temporal preparation improves temporal resolution: Evidence from constant foreperiods. Perception \& Psychophysics, 70, 1504-1514. doi:10.3758/PP.70.8.1504

Boulinguez, P., Jaffard, M., Granjon, L., \& Benraiss, A. (2008). Warning signals induce automatic EMG activations and proactive volitional inhibition: Evidence from analysis of error distribution in simple RT. Journal of Neurophysiology, 99, 1572-1578. doi:10.1152/jn.01198.2007

Boulinguez, P., Ballanger, B., Granjon, L., \& Benraiss, A. (2009). The paradoxical effect of warning on reaction time: Demonstrating proactive response inhibition with event-related potentials. Clinical Neurophysiology, 120, 730-737. doi:10.1016/j.clinph.2009.02.167

Broadbent, D. E., \& Gregory, M. (1965). On the interaction of S-R compatibility with other variables affecting reaction time. British Journal of Psychology, 56, 61-67.

Brunia, C. H. M., \& Boelhouwer, A. H. W. (1988). Reflexes as a tool: A window in the central nervous system. In P. K. Ackles, J. R. Jennings, \& M. G. H. Coles (Eds.), Advances in psychophysiology (Vol. 3, pp. 1-67). Greenwich, CT: JAI Press.

Bueti, D., Bahrami, B., Walsh, V., \& Rees, G. (2010). Encoding of temporal probabilities in the human brain. Journal of Neuroscience, 30, 4343-4352. doi:10.1523/jneurosci.2254-09.2010

Burle, B., Tandonnet, C., \& Hasbroucq, T. (2010). Excitatory and inhibitory motor mechanisms of temporal preparation. In A. C. Nobre \& J. Coull (Eds.), Attention and time (pp. 244-255). Oxford: Oxford Universitly Press.

Bush, L. K., Hess, U., \& Wolford, G. (1993). Transformations for within-subject designs: A Monte Carlo investigation. Psychological Bulletin, 113, 566-579. doi:10.1037/0033-2909.113.3.566

Cardoso-Leite, P., Mamassian, P., \& Gorea, A. (2009). Comparison of perceptual and motor latencies via anticipatory and reactive response times. Attention Perception \& Psychophysics, 71, 8294. doi:10.3758/APP.71.1.82
Correa, Á., Lupiáñez, J., Milliken, B., \& Tudela, P. (2004). Endogenous temporal orienting of attention in detection and discrimination tasks. Perception \& Psychophysics, 66, 264278.

Correa, Á., Lupiáñez, J., \& Tudela, P. (2005). Attentional preparation based on temporal expectancy modulates processing at the perceptual level. Psychonomic Bulletin \& Review, 12, 328-334. doi:10.3758/BF03196380

Correa, Á., Lupiáñez, J., Madrid, E., \& Tudela, P. (2006a). Temporal attention enhances early visual processing: A review and new evidence from event-related potentials. Brain Research, 1076, 116-128. doi:10.1016/j.brainres.2005.11.074

Correa, Á., Lupiáñez, J., \& Tudela, P. (2006b). The attentional mechanism of temporal orienting: Determinants and attributes. Experimental Brain Research, 169, 58-68. doi:10.1007/s00221005-0131-x

Correa, Á., Sanabria, D., Spence, C., Tudela, P., \& Lupiáñez, J. (2006c). Selective temporal attention enhances the temporal resolution of visual perception: Evidence from a temporal order judgment task. Brain Research, 1070, 202-205. doi:10.1016/j. brainres.2005.11.094

Correa, Á., Cappucci, P., Nobre, A. C., \& Lupiáñez, J. (2010a). The two sides of temporal orienting: Facilitating perceptual selection, disrupting response selection. Experimental Psychology, 57, 142148. doi:10.1027/1618-3169/a000018

Correa, Á., Triviño, M., Pérez-Dueñas, C., Acosta, A., \& Lupiáñez, J. (2010b). Temporal preparation, response inhibition and impulsivity. Brain and Cognition, 73, 222-228. doi:10.1016/j. bandc.2010.05.006

Coull, J. T., \& Nobre, A. C. (1998). Where and when to pay attention: The neural systems for directing attention to spatial locations and to time intervals as revealed by both PET and fMRI. Journal of Neuroscience, 18, 7426-7435.

Davranche, K., Tandonnet, C., Burle, B., Meynier, C., Vidal, F., \& Hasbroucq, T. (2007). The dual nature of time preparation: Neural activation and suppression revealed by transcranial magnetic stimulation of the motor cortex. European Journal of Neuroscience, 25, 3766-3774. doi:10.1111/j.1460-9568.2007.05588.x

Duclos, Y., Burnet, H., Schmied, A., \& Rossi-Durand, C. (2008a). Approximate entropy of motorneuron firing patterns during a motor preparation task. Journal of Neuroscience Methods, 172, 231-235. doi:10.1016/j.jneumeth.2008.05.001

Duclos, Y., Schmied, A., Burle, B., Burnet, H., \& Rossi-Durand, C. (2008b). Anticipatory changes in human motoneuron discharge patterns during motor preparation. Journal of Physiology, 586, 1017-1028. doi:10.1113/jphysiol.2007.145318

Duque, J., \& Ivry, R. B. (2009). Role of corticospinal suppression during motor preparation. Cerebral Cortex, 19, 2013-2024. doi:10.1093/cercor/bhn230

Duque, J., Lew, D., Mazzocchio, R., Olivier, E., \& Ivry, R. B. (2010). Evidence for two concurrent inhibitory mechanisms during response preparation. Journal of Neuroscience, 30, 3793-3802. doi:10.1523/jneurosci.5722-09.2010

Elithorn, A., \& Lawrence, C. (1955). Central inhibition: Some refractory observations. Quarterly Journal of Experimental Psychology, 11, 211-220.

Ellis, R. J., \& Jones, M. R. (2010). Rhythmic context modulates foreperiod effects. Attention, Perception, \& Psychophysics, 72, 2274-2288. doi:10.3758/APP.72.8.2274

Fecteau, J. H., \& Munoz, D. P. (2007). Warning signals influence motor processing. Journal of Neurophysiology, 97, 1600-1609. doi:10.1152/jn.00978.2005.

Fischer, R., Plessow, F., \& Kiesel, A. (2010). Auditory warning signals affect mechanisms of response selection: Evidence from a Simon task. Experimental Psychology, 57, 89-97. doi:10.1027/16183169/a000012 
Grondin, S. (2001). From physical time to the first and second moments of psychological time. Psychological Bulletin, 127, 22-44.

Grondin, S., \& Rammsayer, T. (2003). Variable foreperiods and temporal discrimination. Quarterly Journal of Experimental Psychology, 56A, 731-765. doi:10.1080/02724980244000611

Hackley, S. A. (2009). The speeding of voluntary reaction by a warning signal. Psychophysiology, 46, 225-233. doi:10.1111/ j.1469-8986.2008.00716.x

Hackley, S. A., \& Valle-Inclán, F. (1998). Automatic alerting does not speed late motoric processes in a reaction-time task. Nature, 391, 786-788.

Hackley, S. A., \& Valle-Inclán, F. (2003). Which stages of processing are speeded by a warning signal? Biological Psychology, 27-45. doi:10.1016/s0301-0511(03)00101-7

Hackley, S. A., Schankin, A., Wohlschlaeger, A., \& Wascher, E. (2007). Localization of temporal preparation effects via trisected reaction time. Psychophysiology, 44, 334-338. doi:10.1111/ j.1469-8986.2007.00500.x

Haering, C., \& Kiesel, A. (in press). Time in action contexts: Learning when an action effect occurs. Psychological Research. doi:10.1007/s00426-011-0341-8

Jaffard, M., Benraiss, A., Longcamp, M., Velay, J. L., \& Boulinguez, P. (2007). Cueing method biases in visual detection studies. Brain Research, 1179, 106-118. doi:10.1016/j.brainres.2007.08.032

Jaffard, M., Longcamp, M., Velay, J. L., Anton, J.-L., Roth, M., Nazarian, B., et al. (2008). Proactive inhibitory control of movement assessed by event-related fMRI. NeuroImage, 42, $1196-1206$

Janssen, P., \& Shadlen, M. N. (2005). A representation of the hazard rate of elapsed time in macaque area LIP. Nature Neuroscience, 8 , 234-241. doi:10.1038/nn1386

Karlin, L. (1966). Development of readiness to respond during short foreperiods. Journal of Experimental Psychology, 72, 505-509.

Kingstone, A. (1992). Combining expectancies. Quarterly Journal of Experimental Psychology, 44A, 69-104.

Lange, K. (2009). Brain correlates of early auditory processing are attenuated by expectations for time and pitch. Brain and Cognition, 69, 127-137. doi:10.1016/j.bandc.2008.06.004

Lange, K., \& Heil, M. (2008). Temporal attention in the processing of short melodies: Evidence from event-related potentials. Musicae Scientiae, 12, 27-48. doi:10.1177/102986490801200103

Lange, K., \& Röder, B. (2006). Orienting attention to points in time improves stimulus processing both within and across modalities. Journal of Cognitive Neuroscience, 18, 715-729. doi:10.1162/ jocn.2006.18.5.715

Lange, K., Rösler, F., \& Röder, B. (2003). Early processing stages are modulated when auditory stimuli are presented at an attended moment in time: An event-related potential study. Psychophysiology, 40, 806-817. doi:10.1111/1469-8986.00081

Lange, K., Krämer, U. M., \& Röder, B. (2006). Attending points in time and space. Experimental Brain Research, 173, 130-140.

Lohmann, J., Herbort, O., Wagener, A., \& Kiesel, A. (2009). Anticipation of time spans: New data from the foreperiod paradigm and the adaptation of a computational model. In G. Pezzulo, M. V. Butz, O. Sigaud, \& G. Baldassare (Eds.), ABiALS 2008 (pp. 170-187). Berlin: Springer.

Los, S. A., \& Agter, F. (2005). Reweighting sequential effects across different distributions of foreperiods: Segregating elementary contributions to nonspecific preparation. Perception \& Psychophysics, 67, 1161-1170. doi:10.3758/BF03193549

Los, S. A., \& Heslenfeld, D. J. (2005). Intentional and unintentional contributions to nonspecific preparation: Electrophysiological evidence. Journal of Experimental Psychology: General, 134, 52-72. doi:10.1037/0096-3445.134.1.52

Los, S. A., \& Horoufchin, H. (2011). Dissociative patterns of foreperiod effects in temporal discrimination and reaction time tasks. Quarterly Journal of Experimental Psychology, 64, 10091020. doi: $10.1080 / 17470218.2010 .532225$

Los, S. A., \& Schut, M. L. J. (2008). The effective time course of preparation. Cognitive Psychology, 57, 20-55. doi:10.1016/j. cogpsych.2007.11.001

Los, S. A., \& Van den Heuvel, C. E. (2001). Intentional and unintentional contributions to nonspecific preparation during reaction time foreperiods. Journal of Experimental Psychology: Human Perception and Performance, 27, 370-386.

Mattes, S., \& Ulrich, R. (1997). Response force is sensitive to the temporal uncerainty of response stimuli. Perception \& Psychophysics, 59. 1089-1097. doi:10.3758/BF03205523

Mowrer, O. H. (1940). Preparatory set (expectancy)—Some methods of measurement. Psychological Monographs, $52(2$ Whole No. 233), 1-43.

Müller-Gethmann, H., Ulrich, R., \& Rinkenauer, G. (2003). Locus of the effect of temporal preparation: Evidence from the lateralized readiness potential. Psychophysiology, 40, 597-611. doi:10.1111/ $1469-8986.00061$

Näätänen, R. (1970). The diminishing time-uncertainty with the lapse of time after the warning signal in reaction-time experiments with varying fore-periods. Acta Psychologica, 34, 399-418. doi:10.1016/0001-6918(70)90035-1

Nickerson, R. S. (1967). Expectancy, waiting time and the psychological refractory period. Acta Psychologica, 27, 23-34. doi:10.1016/0001-6918(67)90041-8

Niemi, P., \& Näätänen, R. (1981). Foreperiod and simple reaction time. Psychological Bulletin, 89, 133-162. doi:10.1037/00332909.89.1.133

Nobre, A. C., \& Coull, J. T. (Eds.). (2010). Attention and time. Oxford: University Press.

Pecenka, N., \& Keller, P. E. (2009). Auditory pitch imagery and its relationship to musical synchronisation. Annals of the New York Academy of Sciences, 1169, 282-286.

Riehle, A. (2005). Preparation for action: One of the key functions of the motor cortex. In A. Riehle \& E. Vaadia (Eds.), Motor cortex in voluntary movements: A distributed system for distributed functions (pp. 213-240). Boca Raton, FL: CRC Press.

Rimmele, J., Jolsvai, H., \& Sussman, E. (2010). Auditory target detection is affected by implicit temporal and spatial expectations. Journal of Cognitive Neuroscience, 23, 1136-1147.

Rolke, B. (2008). Temporal preparation facilitates perceptual identification of letters. Perception \& Psychophysics, 70, 1305-1313. doi:10.3758/PP.70.7.1305

Rolke, B., \& Hofmann, P. (2007). Temporal uncertainty degrades perceptual processing. Psychonomic Bulletin \& Review, 14, 522 526. doi:10.3758/BF03194101

Sanabria, D., Capizzi, M., \& Correa, Á. (2011). Rhythms that speed you up. Journal of Experimental Psychology: Human Perception and Performance, 37, 236-244. doi:10.1037/a0019956

Sanders, A. F. (1980). Some effects of instructed muscle tension on choice reaction time and movement time. In R. S. Nickerson (Ed.), Attention and performance VIII (pp. 59-74). Hillsdale: Erlbaum.

Sanders, A. F. (1998). Elements of human pefromance: Reaction processes and attention in human skill. Mahwah: Erlbaum.

Schneider, W., Eschman, A., \& Zuccolotto, A. (2002). E-Prime user's guide. Pittsburgh: Psychology Software Tools Inc.

Seibold, V. C., Bausenhart, K. M., Rolke, B., \& Ulrich, R. (2011a). Does temporal preparation increase the rate of sensory information accumulation? Acta Psychologica, 137, 56-64. doi:10.1016/j. actpsy.2011.02.006

Seibold, V. C., Fiedler, A., \& Rolke, B. (2011b). Temporal attention shortens perceptual latency: A temporal prior entry effect. Psychophysiology, 48, 708-717. doi:10.1111/j.14698986.2010.01135.x 
Seifried, T., Ulrich, R., Bausenhart, K. M., Rolke, B., \& Osman, A. (2010). Temporal preparation decreases perceptual latency: Evidence from a clock paradigm. Quarterly Journal of Experimental Psychology, 63, 2432-2451. doi:10.1080/ 17470218.2010 .485354

Sinclair, C., \& Hammond, G. R. (2008). Reduced intracortical inhibition during the foreperiod of a warned reaction time task. Experimental Brain Research, 186, 385-392. doi:10.1007/ s00221-007-1241-4

Sinclair, C., \& Hammond, G. R. (2009). Excitatory and inhibitory processes in primary motor cortex during the foreperiod of a warned reaction time task are unrelated to response expectancy. Experimental Brain Research, 194, 103-113. doi:10.1007/ s00221-008-1684-2

Spijkers, W. A. (1990). The relation between response-specificity, S-R compatibility, foreperiod duration and muscle-tension in a target aiming task. Acta Psychologica, 75, 262-277. doi:10.1016/00016918(90)90016-9

Steinborn, M. B., Rolke, B., Bratzke, D., \& Ulrich, R. (2008). Sequential effects within a short foreperiod context: Evidence for the conditioning account of temporal preparation. Acta Psychologica, 129, 297-307. doi:10.1016/j.actpsy.2008.08.005

Steinborn, M. B., Rolke, B., Bratzke, D., \& Ulrich, R. (2009). Dynamic adjustment of temporal preparation: Shifting warning signal modality attenuates the sequential foreperiod effect. Acta Psychologica, 132, 40-47. doi:10.1016/j.actpsy.2009.06.002

Stuss, D. T., Alexander, M. P., Shallice, T., Picton, T. W., Binns, M. A., Macdonald, R., et al. (2005). Multiple frontal systems controlling response speed. Neuropsychologia, 43, 396-417. doi:10.1016/j. neuropsychologia.2004.06.010

Tandonnet, C., Burle, B., Vidal, F., \& Hasbroucq, T. (2003). The influence of time preparation on motor processes assessed by surface Laplacian estimation. Clinical Neurophysiology, 114, 2376-2384. doi:10.1016/s1388-2457(03)00253-0
Tandonnet, C., Burle, B., Vidal, F., \& Hasbroucq, T. (2006). Knowing when to respond and the efficiency of the cortical motor command: A Laplacian ERP study. Brain Research, 1109, 158163.

Tandonnet, C., Garry, M. I., \& Summers, J. J. (2010). Cortical activation during temporal preparation assessed by transcranial magnetic stimulation. Biological Psychology, 85, 481-486. doi:10.1016/j.biopsycho.2010.08.016

Thomaschke, R., Wagener, A., Kiesel, A., \& Hoffmann, J. (2011). The scope and precision of specific temporal expectancy: Evidence from a variable foreperiod paradigm. Attention Perception \& Psychophysics, 73, 953-964. doi:10.3758/ s13414-010-0079-1

Vallesi, A., Binns, M. A., \& Shallice, T. (2008). An effect of spatialtemporal association of response codes: Understanding the cognitive representations of time. Cognition, 107, 501-527. doi:10.1016/j.cognition.2007.10.011

Van Elswijk, G., Kleine, B. U., Overeem, S., \& Stegeman, D. F. (2007). Expectancy induces dynamic modulation of corticospinal excitability. Journal of Cognitive Neuroscience, 19, 121-131.

Wagener, A., \& Hoffmann, J. (2010a). Behavioural adaptations to redundant frequency distributions in time. In J. Coull \& K. Nobre (Eds.), Attention and time (pp. 217-226). Oxford: Oxford University Press.

Wagener, A., \& Hoffmann, J. (2010b). Temporal cueing of targetidentity and target-location. Experimental Psychology, 57, 436445. doi:10.1027/1618-3169/a000054

Woodrow, H. (1914). The measurement of attention. Psychological Monographs, 17(5), 1-158.

Wundt, W. (1874). Grundzüge der Physiologischen Psychologie. Leipzig: Engelmann.

Zahn, T. P., \& Rosenthal, D. (1966). Simple reaction time as a function of relative frequency of the preparatory interval. Journal of Experimental Psychology, 72, 15-19. doi:10.1037/h0023328 